\title{
CLUSTER VALUES OF HOLOMORPHIC FUNCTIONS OF BOUNDED TYPE
}

RICHARD M. ARON, DANIEL CARANDO, SILVIA LASSALLE, AND MANUEL MAESTRE

\begin{abstract}
We study the cluster value theorem for $H_{b}(X)$, the Fréchet algebra of holomorphic functions bounded on bounded sets of $X$. We also describe the (size of) fibers of the spectrum of $H_{b}(X)$. Our results are rather complete whenever $X$ has an unconditional shrinking basis and for $X=\ell_{1}$. As a byproduct, we obtain results on the spectrum of the algebra of all uniformly continuous holomorphic functions on the ball of $\ell_{1}$.
\end{abstract}

\section{INTRODUCTION}

Our interest in cluster values has its origin in a 1961 paper by a "conglomerate" consisting of the brilliant, albeit fictitious, I. J. Schark Sch61. This work linked the set of cluster points at a boundary point $e^{i \theta}$ of an $H^{\infty}$ function $f$ with the set of evaluations $\varphi(f)$ of elements in the maximal ideal space of $H^{\infty}$ that lie over $e^{i \theta}$. Although I. J. Schark's contribution was soon overshadowed by L. Carleson's paper that solved the corona problem for the disk the following year, ample reasons remain to not dismiss the earlier paper. For one thing, although the corona problem is known to hold for finitely connected domains in $\mathbb{C}$, it is apparently unknown if it holds for all planar domains or for the most standard domains in $\mathbb{C}^{n}, n \geq 2$, such as the bidisk and the ball. Moreover, its higher dimensional analogue is known to be false in general. On the other hand, the authors are unaware of any situation in which the result of I. J. Schark fails.

Indeed, the main objective of this note is to study a version of I. J. Schark's theorem in the context of the Fréchet algebra $H_{b}(X)$ of entire functions of bounded type on a complex Banach space $X$. Thus, this paper builds on the recent paper ACGLM12 of the authors with T. Gamelin, which contains a discussion of cluster values for functions in the Banach algebra $H^{\infty}\left(B_{X}\right)$ of bounded holomorphic functions on the open unit ball of $X$. As we will see, there are very rich cluster set and fiber structures in this context.

For other recent results on the cluster value theorem on $H^{\infty}\left(B_{X}\right)$ we refer to the papers by W. B. Johnson and S. Ortega JohOrt12a and JohOrt12b.

We now review the basic background needed for the rest of this paper. For a Banach space $X$, we use $B_{X}$ (or simply $B$ if there is no risk of confusion) for the

Received by the editors March 26, 2013 and, in revised form, November 14, 2013 and January 11,2014

2010 Mathematics Subject Classification. Primary 46J15, 46E50, $30 \mathrm{H} 05$.

Key words and phrases. Cluster value, analytic functions of bounded type, Banach space, spectrum, fiber.

The first and fourth authors were supported by MICINN Project MTM2011-22417.

The second and third authors were partially supported by CONICET PIP 0624, ANPCyT PICT 2011-1456 and UBACyT Grants 1-218, 1-746, and 20020130100474BA. 
open unit ball of $X$, while $B_{X^{* *}}=B^{* *}$ will denote the open unit ball of $X^{* *}$, the bidual of $X$. Also, $\bar{B}$ and $\bar{B}^{* *}$ will denote the corresponding closed unit balls.

We recall that $H_{b}(X)$ is the algebra of complex valued entire functions on $X$ which are bounded on bounded sets. This is a Fréchet algebra when endowed with the topology of uniform convergence on bounded subsets of $X$. The set of nontrivial $\mathbb{C}$-valued homomorphisms, or spectrum, of this algebra will be denoted by $\mathcal{M}\left(H_{b}(X)\right)$ or $\mathcal{M}$ for short. It is endowed with the weak-star topology $w\left(H_{b}(X)^{*}, H_{b}(X)\right)$. We have the following diagram:

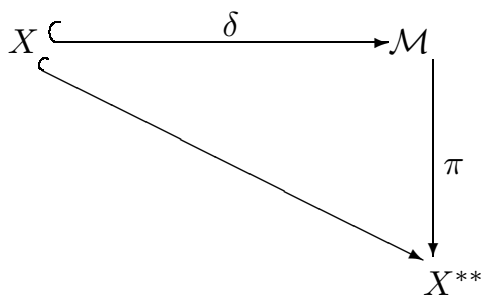

where, as usual, $\delta$ is the point evaluation mapping and $\pi$ is defined by $\pi(\varphi)=$ $\left.\varphi\right|_{X^{*}} \in X^{* *}, \varphi \in \mathcal{M}$.

In general, given an open subset $\Omega$ of a complex Banach space $X$ and a Banach or Fréchet algebra $\mathcal{A}$ of holomorphic functions on $\Omega$, let $\mathcal{M}(\mathcal{A})$ be the set of (continuous) homomorphisms on $\mathcal{A}$. The corona of $\mathcal{M}(\mathcal{A})$ is defined as the set $\mathcal{M}(\mathcal{A}) \backslash{\overline{\left\{\delta_{x}: x \in \Omega\right\}}}^{w *}$. The corona theorem is said to hold whenever the corona is the empty set or, in other words, when the evaluations on points of $\Omega$ form a dense set in $\mathcal{M}(\mathcal{A})$. The corona problem was solved in the affirmative by Carleson Car62 for $H^{\infty}(\mathbb{D})$, where $\mathbb{D}$ is the complex unit disk. Also, if $X$ is finite dimensional, $\mathcal{M}\left(H_{b}(X)\right)=\left\{\delta_{x}: x \in X\right\}$ and the corona theorem holds trivially. For infinite dimensional Banach spaces $X$, the situation is quite different, and it is an open question if the corona theorem is true or not for $H_{b}(X)$ for any $X$. This is why we discuss the cluster value theorem for $H_{b}(X)$ (informally called the weak corona theorem). Even if at first glance the relationship between these two theorems might not be apparent, there is a clear one that can be found in ACGLM12, Lemma 2.3] and in the survey [CGMS12, Proposition 3.8].

The fiber of $\mathcal{M}$ over $z \in X^{* *}$ is the set $\mathcal{M}_{z}:=\pi^{-1}(z)$. In ACG91 it is proved that, for every $z \in X^{* *}$, the fiber $\mathcal{M}_{z}$ is not empty. Indeed, in DavGam89] it is shown that the extension from $H_{b}(X) \rightarrow H_{b}\left(X^{* *}\right)$ exists. It isn't hard to get that it is multiplicative; i.e. the extension morphism

$$
\sim: f \in H_{b}(X) \rightarrow \tilde{f} \in H_{b}\left(X^{* *}\right)
$$

is an algebra homomorphism. Given $z \in X^{* *}$ we define $\tilde{\delta}_{z}: H_{b}(X) \rightarrow \mathbb{C}$ by $\tilde{\delta}_{z}(f)=$ $\tilde{f}(z)$. We have $\tilde{\delta}_{z} \in \mathcal{M}$. If a function $f \in H_{b}(X)$ is weakly continuous when restricted to bounded subsets of $X$, then $\tilde{f}$ is weak-star continuous when restricted to bounded subsets of $X^{* *}$. As a consequence, we also have $\pi\left(\tilde{\delta}_{z}\right)=z$ for every $z \in X^{* *}$.

We remark that the set $\left\{\tilde{\delta}_{z}: z \in X^{* *}\right\}$ is always included in $\overline{\left\{\delta_{x}: x \in X\right\}}$. Indeed, in DavGam89, Theorem 1] it is proved that given $z \in X^{* *}$ there exists a net $\left\{x_{\alpha}\right\}$ in $\|z\| B_{X}$ such that $P\left(x_{\alpha}\right)$ converges to $\tilde{P}(z)$ for every continuous polynomial $P$ on $X$. By the density of polynomials in $H_{b}(X)$, our claim follows. The analogous 
result for $\mathcal{A}=H^{\infty}\left(B_{X}\right)$ or the ball algebra (defined below) $\mathcal{A}=A_{u}\left(B_{X}\right)$ follows from [DavGam89, Lemma on p. 355].

Following [ACG91, the radius function on $\mathcal{M}$ is defined by

$$
R(\varphi)=\inf \left\{r>0:|\varphi(f)| \leq\|f\|_{r B}\right\},
$$

where $\|f\|_{r B}$ is the supremum of $|f|$ over $r B$. For $\|z\| \leq r$, we also define the sets

$$
\begin{gathered}
\mathcal{M}_{z, r}=\mathcal{M}_{z, r}\left(H_{b}(X)\right)=\left\{\varphi \in \mathcal{M}_{z}: R(\varphi) \leq r\right\} \text { and } \\
\mathcal{M}_{r}=\mathcal{M}_{r}\left(H_{b}(X)\right)=\{\varphi \in \mathcal{M}: R(\varphi) \leq r\} .
\end{gathered}
$$

We will also consider the ball algebra $A_{u}(r B)$, which is the Banach algebra of all uniformly continuous holomorphic functions on $r B$. The spectrum of $A_{u}(r B)$ will be denoted by $\mathcal{M}\left(A_{u}(r B)\right)$ and is a compact set endowed with the weak-star topology $w\left(A_{u}(r B)^{*}, A_{u}(r B)\right)$. We denote the corresponding fiber over $z \in r \bar{B}^{* *}$ by $\mathcal{M}_{z}\left(A_{u}(r B)\right)$.

In ACG91, Section 12] it is noted that $H_{b}(X)$ can be embedded into $A_{u}(r B)$ by the restriction mapping, and a relationship between the spectra of these algebras is presented. As this fact will be crucial in our study of $\mathcal{M}\left(H_{b}(X)\right)$ and to make this paper self-contained, we will give a proof of it in Lemma 1.2

Let us now introduce the main topic of this work. For fixed $r>0, f$ a holomorphic function bounded on $r B$, and $z_{0} \in X^{* *}$ with $\left\|z_{0}\right\| \leq r$, we define the $r$-cluster set of $f$ at $z_{0}$ as the set $C l_{r}\left(f, z_{0}\right)$ of all limits of values of $f$ along nets in $r B$ converging weak-star to $z_{0}$. In the particular case that $z_{0}$ actually belongs to $r B$, $f\left(z_{0}\right)$ belongs to $C l_{r}\left(f, z_{0}\right)$. Moreover, if $z_{0}$ belongs to $r B^{* *}$, then $\tilde{f}\left(z_{0}\right)$ belongs to $C l_{r}\left(f, z_{0}\right)$ for any $f \in H_{b}(X)$. Indeed, for polynomials, this is a direct consequence of Davie and Gamelin's result mentioned above (Theorem 1 in DavGam89]). Since functions in $H_{b}(X)$ are uniform limits of polynomials on bounded sets, the conclusion follows.

Following [ACGLM12, we simply write $C l\left(f, z_{0}\right)$ for $C l_{1}\left(f, z_{0}\right)$ (that is, when $z_{0}$ and all the considered nets belong to the corresponding unit balls). Classical spaces $\ell_{p}$ for $1 \leq p<\infty$ give examples of cluster sets that are not trivial (see Proposition 2.6).

The cluster set $C l_{r}\left(f, z_{0}\right)$ can be seen as the intersection of the closures of $f(U \cap r B)$, where $U$ ranges over any basis for the weak-star neighborhoods of $z_{0}$. Choosing a basis of convex sets, we see that $C l_{r}\left(f, z_{0}\right)$ is an intersection of a decreasing net $\left(K_{\alpha}\right)_{\alpha}$ of nonempty compact connected sets. As a consequence (see, for instance, Exercise 11 in [Munkres, Section 26]), each cluster set $C l_{r}\left(f, z_{0}\right)$, $z_{0} \in r \bar{B}^{* *}$, is a nonempty compact connected set. Actually we also have that $C l_{r}\left(f, z_{0}\right)$ coincides with the set

$$
\left\{\lambda \in \mathbb{C}: \text { there exists }\left(z_{\alpha}\right) \subset r B^{* *} \text { such that } z_{\alpha} \stackrel{w\left(X^{* *}, X^{*}\right)}{\longrightarrow} z_{0} \text { and } \tilde{f}\left(z_{\alpha}\right) \rightarrow \lambda\right\} .
$$

Indeed, let us call this last set $C$. Clearly $C l_{r}\left(f, z_{0}\right)$ is contained in $C$. On the other hand $C=\bigcap_{U \in \mathcal{U}} \overline{\tilde{f}\left(U \cap r B^{* *}\right)}$, where $\mathcal{U}$ is a basis of open convex weak-star neighborhoods of 0. But, by [DavGam89, Lemma of Theorem 5], $\tilde{f}\left(U \cap r B^{* *}\right)$ (and hence $\left.\bar{f}\left(U \cap r B^{* *}\right)\right)$ is contained in $\overline{f(U \cap r B)}$ for every $U$ in $\mathcal{U}$ and we have

$$
C l_{r}\left(f, z_{0}\right) \subset C=\bigcap_{U \in \mathcal{U}} \overline{\tilde{f}\left(U \cap r B^{* *}\right)} \subset \bigcap_{U \in \mathcal{U}} \overline{f(U \cap r B)}=C l_{r}\left(f, z_{0}\right) .
$$

We refer to ColLoh66 for more information on cluster sets and their properties. 
As in Lemma 2.2 of ACGLM12, we always have

$$
C l_{r}\left(f, z_{0}\right) \subset \hat{f}\left(\mathcal{M}_{z_{0}, r}\right),
$$

where $\hat{f}: \mathcal{M} \rightarrow \mathbb{C}$ is the Gel'fand transform of $f$ given by $\hat{f}(\varphi)=\varphi(f)$. Therefore, we are interested in the reverse inclusion. When the equality holds we say that we have a cluster value theorem at $z_{0}$. This has been very recently studied in ACGLM12 for the case of the Banach algebra $A_{u}\left(r B_{X}\right)$.

In Section 1, we prove a version of a weak corona theorem for $H_{b}(X)$. Although providing set inclusions rather than a set equality as in Theorems 4.1 and 5.1 of ACGLM12, our results hold in much more generality. Section 2 is devoted to characterizing point evaluations $\delta_{x}$ among all the homomorphisms in $\mathcal{M}_{x}$, while the final section deals with a closer examination of the fiber structure of $\mathcal{M}\left(A_{u}\left(B_{\ell_{1}}\right)\right)$.

For background on Banach spaces, see [Die84]. For background on analytic functions on Banach spaces, see Din99], Gam94 or Muj86. For background on uniform algebras, see Gam69.

\section{The Cluster value theorem For $H_{b}(X)$}

We recall that given a Banach space $X, P_{f}(X)$ is the unital subalgebra of the normed algebra of continuous polynomials on $X$ generated by the monomials $x \mapsto$ $x_{1}^{*}(x) \cdots x_{n}^{*}(x)$, where $x_{j}^{*} \in X^{*}, 1 \leq j \leq n$, and $n \in \mathbb{N}$. Polynomials in the closure of $P_{f}(X)$ are called approximable polynomials. It is well-known, and immediate to check, that $\varphi(P)=\tilde{\delta}_{\pi(\varphi)}(P)$ for every approximable polynomial $P$ and every homomorphism $\varphi$ in $\mathcal{M}\left(H_{b}(X)\right)$. Hence, given a complex Banach space $X$ such that all continuous polynomials are approximable, $\mathcal{M}\left(H_{b}(X)\right)=\left\{\tilde{\delta}_{z}: z \in X^{* *}\right\}$. In this case, the cluster value theorem trivially holds for $H_{b}(X)$.

Proposition 1.1. Let $X$ be a complex Banach space such that all continuous polynomials are approximable. Then

$$
C l_{r}(f, z)=\hat{f}\left(\mathcal{M}_{z, r}\right),
$$

for every $f \in H_{b}(X)$, every $r>0$ and $z \in X^{* *}$ with $\|z\| \leq r$.

Spaces in which all polynomials are approximable are, for example, $c_{0}$, the original Tsirelson space $T^{*}$ and the Tsirelson-James space $T_{J}^{*}$.

Under certain hypotheses, cluster value theorems of the form

$$
C l_{r}(g, z)=\hat{g}\left(\mathcal{M}_{z}\left(A_{u}\left(r B_{X}\right)\right)\right), \quad g \in A_{u}\left(r B_{X}\right),
$$

for $z \in r B^{* *}$, were proved in ACGLM12. The following two lemmas allow us to transfer these results to $H_{b}(X)$.

Lemma 1.2 ([ACG91, Section 12]). Let $i: H_{b}(X) \rightarrow A_{u}(r B),\left.f \rightsquigarrow f\right|_{r B}$, be the natural inclusion mapping and let $i^{t}: A_{u}(r B)^{*} \rightarrow H_{b}(X)^{*}$ be its transpose. If we denote by $\Phi$ the restriction of $i^{t}$ to $\mathcal{M}\left(A_{u}(r B)\right)$, then $\Phi: \mathcal{M}\left(A_{u}(r B)\right) \rightarrow \mathcal{M}_{r}\left(H_{b}(X)\right)$ is a homeomorphism onto, for every $r>0$.

Proof. Clearly $\Phi$ is well defined and continuous. By the Alaoglu-Bourbaki Theorem both sets $\mathcal{M}\left(A_{u}(r B)\right)$ and $\mathcal{M}_{r}\left(H_{b}(X)\right)$ are compact. Hence, to obtain the conclusion it is enough to check that $\Phi$ is a bijective mapping. Consider $\varphi \in \mathcal{M}\left(A_{u}(r B)\right)$ and $f \in H_{b}(X)$. Then $\Phi(\varphi)(f)=\varphi\left(\left.f\right|_{r B}\right)$, where $\left.f\right|_{r B}$ stands for the restriction of $f$ to $r B$. Hence, from the well-known fact that the set of all continuous polynomials on $X$ is dense on $A_{u}(r B)$ we obtain that $\Phi$ is an injective mapping. Now 
consider $\eta \in \mathcal{M}_{r}\left(H_{b}(X)\right)$. By hypothesis, $|\eta(f)| \leq\|f\|_{r}$ for every $f \in H_{b}(X)$. Fix $g \in A_{u}(r B)$ and consider a sequence of polynomials $\left(P_{n}\right)_{n}$ that is uniformly convergent to $g$ on $r B$. Since $\left|\eta\left(P_{n}-P_{m}\right)\right| \leq\left\|P_{m}-P_{m}\right\|_{r}$ for every $n, m \in \mathbb{N}$, we get that $\left(\eta\left(P_{n}\right)\right)_{n}$ is a Cauchy sequence in $\mathbb{C}$. Define

$$
\varphi(g)=\lim _{n} \eta\left(P_{n}\right) .
$$

It is not difficult to check that $\varphi \in \mathcal{M}\left(A_{u}(r B)\right)$. Since polynomials are dense in $H_{b}(X)$, we conclude that $\Phi(\varphi)=\eta$.

Lemma 1.3. Let $X$ be a Banach space and suppose that the cluster value theorem (2) holds at some $z_{0} \in X^{* *}$ with $\left\|z_{0}\right\| \leq r$. Then,

$$
C l_{r}\left(f, z_{0}\right)=\hat{f}\left(\mathcal{M}_{z_{0}, r}\right) \quad \text { for every } f \in H_{b}(X) .
$$

Proof. One inclusion is (1). For the converse, fix $\varphi \in \mathcal{M}_{z_{0}, r}$. By Lemma 1.2, we know that

$$
\{\psi \in \mathcal{M}: R(\psi) \leq r\}=\mathcal{M}\left(A_{u}(r B)\right) .
$$

Then there exists $\bar{\varphi} \in \mathcal{M}\left(A_{u}(r B)\right)$ such that $\varphi(f)=\bar{\varphi}\left(\left.f\right|_{r B}\right)$, for all $f \in H_{b}(X)$.

But our hypotheses then say that

$$
\varphi(f)=\bar{\varphi}\left(\left.f\right|_{r B}\right) \in C l_{r}\left(\left.f\right|_{r B}, z_{0}\right)=C l_{r}\left(f, z_{0}\right),
$$

which is the desired inclusion.

In ACGLM12, Theorem 3.1] it is shown that (2) holds at 0 whenever $X$ is a Banach space with a shrinking 1-unconditional basis. Moreover for $X=\ell_{2},(2)$ holds for every $x \in B_{\ell_{2}}$. The results in [ACGLM12] are for the unit ball, but they clearly hold for balls of any other radius. As a consequence of these results, we have the following.

Theorem 1.4. (a) If $X$ is a Banach space with a shrinking 1-unconditional basis, then

$$
C l_{r}(f, 0)=\hat{f}\left(\mathcal{M}_{0, r}\right) \quad \text { for every } f \in H_{b}(X) .
$$

(b) For $X=\ell_{2}$ we have

$$
C l_{r}\left(f, z_{0}\right)=\hat{f}\left(\mathcal{M}_{z_{0}, r}\right) \quad \text { for every } f \in H_{b}\left(\ell_{2}\right) \text {, all } z_{0} \in r B_{\ell_{2}} \text { and all } r>0 \text {. }
$$

In order to see what happens outside the origin for more general Banach spaces, we first need some lemmas.

Lemma 1.5. Let $x^{*} \in X^{*}$ be a nonzero continuous linear functional and take $e \in X$ such that $x^{*}(e)=1$. For $z \in X^{* *}$ and $f \in H_{b}(X)$, there exists $g \in H_{b}(X)$ such that

$$
f(x)=f\left(x+\left(z\left(x^{*}\right)-x^{*}(x)\right) e\right)+\left(x^{*}(x)-z\left(x^{*}\right)\right) g(x)
$$

for all $x \in X$.

Proof. Let $f=\sum_{n=0}^{\infty} P_{n}$ be the Taylor series expansion of $f$ at 0 , and denote by $A_{n}$ the symmetric $n$-linear mapping associated to $P_{n}$. We have

$$
\begin{aligned}
f(x) & =f\left(x+z\left(x^{*}\right) e-x^{*}(x) e+\left(x^{*}(x)-z\left(x^{*}\right)\right) e\right) \\
& =\sum_{n=0}^{\infty} \sum_{k=0}^{n}\left(\begin{array}{l}
n \\
k
\end{array}\right) A_{n}\left(\left(x-x^{*}(x) e+z\left(x^{*}\right) e\right)^{n-k},\left(x^{*}(x) e-z\left(x^{*}\right) e\right)^{k}\right) .
\end{aligned}
$$


This series converges absolutely. Indeed take $R>1$. For $\|v\| \leq R$ and $\|w\| \leq R$ we have

$$
\begin{aligned}
\sum_{n=0}^{\infty} \sum_{k=1}^{n}\left|\left(\begin{array}{l}
n \\
k
\end{array}\right) A_{n}\left(v^{n-k}, w^{k-1}, e\right)\right| & \leq \sum_{n=0}^{\infty} \sum_{k=1}^{n}\left(\begin{array}{l}
n \\
k
\end{array}\right)\left\|A_{n}\right\|\|v\|^{n-k}\|w\|^{k-1}\|e\| \\
& \leq\|e\| \sum_{n=0}^{\infty}\left\|A_{n}\right\| R^{n-1} \sum_{k=1}^{n}\left(\begin{array}{l}
n \\
k
\end{array}\right) \\
& \leq\|e\| \sum_{n=0}^{\infty}\left\|A_{n}\right\|(2 R)^{n}
\end{aligned}
$$

which is finite since limsup $\sqrt[n]{\left\|A_{n}\right\|}=0$. Therefore, we can rearrange the terms with $k=0$ to obtain

$$
\begin{aligned}
f(x)= & \sum_{n=0}^{\infty} A_{n}\left(\left(x-x^{*}(x) e+z\left(x^{*}\right) e\right)^{n}\right) \\
& +\sum_{n=0}^{\infty} \sum_{k=1}^{n}\left(\begin{array}{l}
n \\
k
\end{array}\right) A_{n}\left(\left(x-x^{*}(x) e+z\left(x^{*}\right) e\right)^{n-k},\left(x^{*}(x) e-z\left(x^{*}\right) e\right)^{k}\right) \\
= & f\left(x-x^{*}(x) e+z\left(x^{*}\right) e\right)+\left(x^{*}(x)-z\left(x^{*}\right)\right) \\
& \times \sum_{n=0}^{\infty} \sum_{k=1}^{n}\left(\begin{array}{l}
n \\
k
\end{array}\right) A_{n}\left(\left(x-x^{*}(x) e+z\left(x^{*}\right) e\right)^{n-k},\left(x^{*}(x) e-z\left(x^{*}\right) e\right)^{k-1}, e\right) .
\end{aligned}
$$

So we have to check that the double sum defines a function in $H_{b}(X)$. For this, we consider the mapping $G: X \times X \rightarrow \mathbb{C}$ given by

$$
G(v, w)=\sum_{n=0}^{\infty} \sum_{k=1}^{n}\left(\begin{array}{l}
n \\
k
\end{array}\right) A_{n}\left(v^{n-k}, w^{k-1}, e\right) .
$$

By (44), the double series defining $G$ converges absolutely and uniformly on bounded subsets of $X \times X$. Each term belongs to $H_{b}(X \times X)$, and then so does $G$. Now, the function $g(x)=G\left(x-x^{*}(x) e+z\left(x^{*}\right) e, x^{*}(x) e-z\left(x^{*}\right) e\right)$ belongs to $H_{b}(X)$ and is the function we are looking for.

Lemma 1.6. Let $X$ be a Banach space with Schauder basis $\left\{e_{k}\right\}_{k}$, and denote by $\left\{e_{k}^{*}\right\}_{k}$ its dual basic sequence. Fix $z \in X^{* *}$. For $f \in H_{b}(X)$ and $N \in \mathbb{N}$, there exist $g_{1}, \ldots, g_{N} \in H_{b}(X)$ such that

$$
f(x)=f\left(\sum_{k=1}^{N} z\left(e_{k}^{*}\right) e_{k}+\sum_{k=N+1}^{\infty} e_{k}^{*}(x) e_{k}\right)+\sum_{k=1}^{N}\left(e_{k}^{*}(x)-z\left(e_{k}^{*}\right)\right) g_{k}(x)
$$

for all $x \in X$.

Proof. Note that

$$
\sum_{k=1}^{N} z\left(e_{k}^{*}\right) e_{k}+\sum_{k=N+1}^{\infty} e_{k}^{*}(x) e_{k}=x+\sum_{k=1}^{N}\left(z\left(e_{k}^{*}\right)-e_{k}^{*}(x)\right) e_{k} .
$$

So the result follows from repeated application of Lemma 1.5

The two preceding results yield the following lemma, which essentially says that if $\varphi$ lies in the fiber above $z$, then $\varphi(f)$ is independent of the first coordinates of $f$. 
Lemma 1.7. Under the same hypotheses as in Lemma 1.6, if $\varphi \in \mathcal{M}_{z}$, then

$$
\varphi(f)=\varphi\left(x \mapsto f\left(\sum_{k=1}^{N} z\left(e_{k}^{*}\right) e_{k}+\sum_{k=N+1}^{\infty} e_{k}^{*}(x) e_{k}\right)\right)
$$

for all $f \in H_{b}(X)$.

Proof. Since $\varphi \in \mathcal{M}_{z}$, we have $\varphi\left(e_{k}^{*}\right)=z\left(e_{k}^{*}\right)$ for all $k$. Writing $f$ as in Lemma 1.6 we obtain the conclusion.

Now we are ready to show our cluster value theorem for Banach spaces with unconditional shrinking bases.

Theorem 1.8. Let $X$ be a Banach space with a shrinking $K$-unconditional basis. Then

$$
C l_{r}\left(f, z_{0}\right) \subset \hat{f}\left(\mathcal{M}_{z_{0}, r}\right) \subset C l_{K\left(\left\|z_{0}\right\|+r\right)}\left(f, z_{0}\right)
$$

for all $f \in H_{b}(X)$ and $z_{0} \in X^{* *}$ with $\left\|z_{0}\right\| \leq r$.

Proof. The first inclusion is (1). For the second one, it is enough to show that if $0 \notin$ $C l_{K\left(\left\|z_{0}\right\|+r\right)}\left(f, z_{0}\right)$, then $0 \notin \hat{f}\left(\mathcal{M}_{z_{0}, r}\right)$. Let $\left\{e_{k}\right\}_{k}$ be the shrinking $K$-unconditional basis of $X$, and denote by $\left\{e_{k}^{*}\right\}_{k}$ its dual basis (which is a $K$-unconditional basis of $\left.X^{*}\right)$. Since the Schauder basis is shrinking, the sets

$$
W_{n, l}=\left\{z \in X^{* *}:\|z\| \leq K\left(\left\|z_{0}\right\|+r\right),\left|z\left(e_{k}^{*}\right)-z_{0}\left(e_{k}^{*}\right)\right|<\frac{1}{l}, k=1, \ldots, n\right\}
$$

form a basis of weak-star neighborhoods of $z_{0}$ in $K\left(\left\|z_{0}\right\|+r\right) B^{* *}$. Therefore, if 0 does not belong to $C l_{K\left(\left\|z_{0}\right\|+r\right)}\left(f, z_{0}\right)$, we can choose $N, p \in \mathbb{N}$ and $\delta>0$ such that $|f|>\delta$ on $W_{N, p}$.

Note that for $x \in r B$ we have

$$
\left\|\sum_{k=1}^{N} z_{0}\left(e_{k}^{*}\right) e_{k}+\sum_{k=N+1}^{\infty} e_{k}^{*}(x) e_{k}\right\| \leq K\left\|z_{0}\right\|+K\|x\| \leq K\left(\left\|z_{0}\right\|+r\right)
$$

and also

$$
e_{j}^{*}\left(\sum_{k=1}^{N} z_{0}\left(e_{k}^{*}\right) e_{k}+\sum_{k=N+1}^{\infty} e_{k}^{*}(x) e_{k}\right)=z_{0}\left(e_{j}^{*}\right) \quad \text { for } j=1, \ldots, N .
$$

This means that $\sum_{k=1}^{N} z_{0}\left(e_{k}^{*}\right) e_{k}+\sum_{k=N+1}^{\infty} e_{k}^{*}(x) e_{k}$ belongs to $W_{N, p}$ for every $x \in$ $r B$. As a consequence, if we define $f_{N}: X \rightarrow \mathbb{C}$ as

$$
f_{N}(x)=f\left(\sum_{k=1}^{N} z_{0}\left(e_{k}^{*}\right) e_{k}+\sum_{k=N+1}^{\infty} e_{k}^{*}(x) e_{k}\right),
$$

it follows that $\left|f_{N}\right|>\delta$ on $r B$ and, as a consequence, $\left.f_{N}\right|_{r B}$ is invertible in $A_{u}(r B)$.

Given any $\varphi \in \mathcal{M}_{z_{0}, r}$, by Lemma 1.2, there exists $\bar{\varphi} \in \mathcal{M}\left(A_{u}(r B)\right)$ such that $\varphi(g)=\bar{\varphi}\left(\left.g\right|_{r B}\right)$, for all $g \in H_{b}(X)$. We combine this with Lemma 1.7 and obtain

$$
\varphi(f)=\varphi\left(f_{N}\right)=\bar{\varphi}\left(\left.f_{N}\right|_{r B}\right) \neq 0,
$$

since $\left.f_{N}\right|_{r B}$ is invertible. Therefore, $0 \notin \hat{f}\left(\mathcal{M}_{z_{0}, r}\right)$ and the proof is finished. 
The above proof is a particular instance involving Banach spaces having separable duals. For results about cluster sets in this situation, it is enough to take sequences rather than nets. Note that, in particular, for Banach spaces with a shrinking 1-unconditional basis, we have a new proof of Theorem 1.4 (a):

$$
C l_{r}(f, 0)=\hat{f}\left(\mathcal{M}_{0, r}\right) \quad \text { for every } f \in H_{b}(X) .
$$

Remark 1.9. A direct consequence of the previous theorem is that

$$
C l_{r}\left(f, z_{0}\right) \subset \hat{f}\left(\mathcal{M}_{z_{0}, r}\right) \subset C l_{2 K r}\left(f, z_{0}\right)
$$

for all $f \in H_{b}(X)$ and $z_{0} \in X^{* *}$ with $\left\|z_{0}\right\| \leq r$. This is a weaker statement, but it is somehow "cleaner": the size of the nets involved in the cluster sets does not depend on $z_{0}$.

For the case $X=\ell_{p}$, we can improve inequality (마) to

$$
\left\|\sum_{k=1}^{N} z_{0}\left(e_{k}^{*}\right) e_{k}+\sum_{k=N+1}^{\infty} e_{k}^{*}(x) e_{k}\right\| \leq \sqrt[p]{\left\|z_{0}\right\|^{p}+r^{p}} .
$$

As a consequence of this, we get

$$
C l_{r}\left(f, z_{0}\right) \subset \hat{f}\left(\mathcal{M}_{z_{0}, r}\right) \subset C l_{\sqrt[p]{\left\|z_{0}\right\|^{p}+r^{p}}}\left(f, z_{0}\right) \subset C l_{\sqrt[p]{2} r}\left(f, z_{0}\right)
$$

for all $f \in H_{b}\left(\ell_{p}\right)$ and $z_{0} \in r B_{\ell_{p}}$. Note, however, that for $\ell_{2}$ this result is weaker than Theorem 1.4 (b). For $c_{0}$ we just reobtain Proposition 1.1 .

\section{Characterizing evaluations}

Evaluations are distinguished elements in the spectrum (see the diagram in the introduction). In this section, we present an intrinsic property that characterizes, for uniformly convex spaces, evaluations among general homomorphisms.

We first recall some results from ACGLM12, which require some definitions that we introduce here. We will denote by $A(B)$ the algebra of uniform limits on $B$ of polynomials in the functions in $X^{*}$; that is, $A(B)$ is the completion of the algebra $P_{f}(X)$ endowed with the sup-norm on $B$. Polynomials in functions in $X^{*}$ extend to be weak-star continuous on the closed unit ball $\bar{B}^{* *}$, and so do their uniform limits. Then, we can consider $A(B)$ as a uniform algebra of continuous functions on $\bar{B}^{* *}$, with the weak-star topology. The functions in $A(B)$ are analytic on $B$. Each nonzero complex-valued homomorphism of $A(B)$ is the evaluation homomorphism at some point of $\bar{B}^{* *}$. Therefore, the spectrum $\mathcal{M}_{A(B)}$ of $A(B)$ coincides with $\bar{B}^{* *}$. We say that a point $x \in \bar{B}^{* *}$ is a peak point for $A(B)$ if there is $g \in A(B)$ such that $g(x)=1$ and $|g(y)|<1$ for $y \in \bar{B}^{* *}, y \neq x$. The function $g$ is said to peak at $x$ (see Gam69]). In the following lemma, $\mathcal{H}$ is any algebra of bounded analytic functions on $B$ containing $A(B)$ and closed under the sup-norm on $B$ (i.e, under the topology of uniform convergence).

Lemma 2.1 (ACGLM12, Lemma 2.4]). Let $x \in \bar{B}$, and suppose that $g$ is a function in $A(B)$ such that $g(x)=1$, while $|g|$ is bounded by a constant strictly less than 1 on any subset of $B$ at a positive distance from $x$. Then $g$ peaks at $x$. Further, if $f \in \mathcal{H}$ and $\lambda \in \mathbb{C}$ are such that $f(y) \rightarrow \lambda$ whenever $y \in B$ tends to $x$ in norm, then $\widehat{f} \equiv \lambda$ on $\mathcal{M}_{x}(\mathcal{H})$.

The following results characterize evaluations among all the elements in the spectrum of $H_{b}(X)$. 
Theorem 2.2. Let $X$ be a Banach space and $x \in X$ such that $x /\|x\|$ is a peak point for $A(B)$. Then, for $\varphi \in \mathcal{M}_{x}$ we have $\varphi=\delta_{x}$ if and only if $R(\varphi) \leq\|x\|$.

Proof. If $\varphi=\delta_{x}$, then $R(\varphi)=\|x\|$. For the converse, by ACG91, 3.2 Lemma] we always have $R(\varphi) \geq\|x\|$ and then $R(\varphi)=\|x\|$. Without loss of generality we may suppose that $R(\varphi)=\|x\|=1$. We appeal again to Lemma 1.2 to obtain $\bar{\varphi} \in \mathcal{M}\left(A_{u}(B)\right)$ such that $\varphi(f)=\bar{\varphi}\left(\left.f\right|_{B}\right)$, for all $f \in H_{b}(X)$. Now, $\left.f\right|_{B}(y) \rightarrow f(x)$ whenever $y$ tends to $x$ in norm (since $f$ is continuous on $X$ ), so we apply Lemma 2.1 to get

$$
\varphi(f)=\bar{\varphi}\left(\left.f\right|_{B}\right)=f(x) .
$$

This holds for any $f \in H_{b}(X)$ and then $\varphi=\delta_{x}$.

In [Far98, Proposition 4.1], Farmer shows that if $X$ is a uniformly convex Banach space, then for any $x \in S_{X}$ there exists a function $g$ satisfying the hypotheses of Lemma 2.1. As a consequence, every element in $S_{X}$ is a peak point for $A(B)$. Since uniformly convex Banach spaces are reflexive, we obtain the following.

Corollary 2.3. Let $X$ be a uniformly convex Banach space and take $\varphi \in \mathcal{M}$. Then, $\varphi$ is an evaluation if and only if $R(\varphi) \leq\|\pi(\varphi)\|$.

In [AcoLou07, Theorem 2.6] it is proved that any point of the unit sphere of $\ell_{1}$ is a peak point for $A\left(B_{\ell_{1}}\right)$. Also, in Theorem 2.4 of the same paper, the authors show that every point on the unit sphere of the Lorentz sequence space $d(w, 1)$ is a peak point for $A\left(B_{d(w, 1)}\right)$, provided the weight $w$ is in $c_{0} \backslash \ell_{1}$ and $w_{1}=1$. As a consequence, we also have the following.

Corollary 2.4. Let $X$ be $\ell_{1}$ or $d(w, 1)$. Given $\varphi \in \mathcal{M}$ with $\pi(\varphi) \in X$, we have $\varphi=\delta_{\pi(\varphi)}$ if and only if $R(\varphi) \leq\|\pi(\varphi)\|$.

There is another way to state Theorem 2.2 or Corollaries 2.3 and 2.4 . For $X$ under the corresponding hypotheses and $x \in X$, we have

$$
\mathcal{M}_{x,\|x\|}=\left\{\delta_{x}\right\}
$$

Let us see now that we cannot extend Corollary 2.4 to homomorphisms $\varphi$ over arbitrary elements in the bidual. In Section 3 we will study the fibers of the spectrum of $A_{u}\left(B_{\ell_{1}}\right)$. In particular, we will show that there are plenty of elements $z$ in the unit sphere of $\ell_{1}^{* *}$ such that the fiber for $A_{u}\left(B_{\ell_{1}}\right)$ over $z$ is huge.

Using again the result in ACG91, Section 12] stated in Lemma 1.2, we would get (a lot of) $\varphi \in \mathcal{M}$ such that $R(\varphi)=1=\|\pi(\varphi)\|$ but $\varphi$ is not the "evaluation" at $\pi(\varphi)$. However, we prefer to give a direct argument to show the existence of such a $\varphi$.

Proposition 2.5. Let $X$ be $\ell_{1}$. There exists $\varphi \in \mathcal{M}$ such that $R(\varphi)=\|\pi(\varphi)\|$ but $\varphi \neq \tilde{\delta}_{\pi(\varphi)}$.

Proof. Let $\psi$ be an accumulation point of the sequence $\left(\delta_{e_{n}}\right)_{n=1}^{\infty} \in \mathcal{M}\left(A_{u}\left(B_{\ell_{1}}\right)\right)$. Consider its restriction $\varphi$ to $H_{b}\left(\ell_{1}\right)$ and take $z=\pi(\varphi) \in \ell_{1}^{* *}$. Clearly, $R(\varphi) \leq 1$ and then $\|z\| \leq 1$. On the other hand, consider the element $\overrightarrow{1}=(1, \ldots, 1, \ldots) \in \ell_{1}^{*}$. We have

$$
\overrightarrow{1}\left(e_{n}\right)=1 \quad \text { for all } n \text { and then } z(\overrightarrow{1})=\varphi(\overrightarrow{1})=1 \text {. }
$$


As a consequence $\|z\|=1=R(\varphi)$. We claim that $\varphi \neq \tilde{\delta}_{z}$. To show this, we prove that $\varphi$ and $\tilde{\delta}_{z}$ take different values on the polynomial

$$
P(x)=\sum_{k=0}^{\infty} x_{k}^{2}=\sum_{k=0}^{\infty} e_{k}^{*}(x)^{2} .
$$

Since $P\left(e_{n}\right)=1$ for every $n \in \mathbb{N}$, we have $\varphi(P)=1$.

On the other hand, since the symmetric bilinear form $A: \ell_{1} \times \ell_{1} \longrightarrow \mathbb{C}$ associated to $P$ is given by $A(x, y)=\sum_{k=0}^{\infty} x_{k} y_{k}=\sum_{k=0}^{\infty} e_{k}^{*}(x) e_{k}^{*}(y)$, we have $A\left(e_{m}, e_{n}\right)=0$ for all $n>m$. Hence $\lim _{m} \lim _{n} A\left(e_{m}, e_{n}\right)=0$. As a consequence,

$$
\tilde{\delta}_{z}(P)=\tilde{P}(z)=\tilde{A}(z, z)=0 \neq \varphi(P),
$$

and the conclusion follows.

The results in Theorem 2.2 and Corollaries 2.3 and 2.4 give conditions under which there is just one homomorphism in the fiber over $x \in X$ with $R(\varphi)=\|x\|$. This does not mean that all fibers are small. In fact, in general, fibers are rather big. Again, it will help us to see what happens in $A_{u}(B)$.

Proposition 2.6. Let $X$ be a Banach space which admits a homogeneous continuous polynomial whose restriction to the open unit ball is not weakly continuous at 0 . Then, $\operatorname{Card}\left(\mathcal{M}_{z}\left(A_{u}\left(B_{X}\right)\right)\right) \geq c$ for every $z \in B_{X^{* *}}$, where $c$ stands for the cardinal of the continuum.

Proof. Let $P$ be a homogeneous continuous polynomial whose restriction to the open unit ball is not weakly continuous at 0 and fix $z$ in $B_{X^{* *}}$. The proof is in two parts. First, we show that the conclusion holds if there is a homomorphism $\varphi \in \mathcal{M}_{z}\left(A_{u}\left(B_{X}\right)\right)$ and a net $\left(z_{\alpha}\right)$ in $B_{X^{* *}}$ such that $\left(\tilde{\delta}_{z_{\alpha}}\right)$ converges to $\varphi$ and $\left|\tilde{P}\left(z_{\alpha}\right)-\tilde{P}(z)\right|>\varepsilon$, for some $\varepsilon>0$ and for all $\alpha$. Second, we show the existence of $\varphi$ and the net $\left(z_{\alpha}\right)$.

For the first assertion, note that for each $x^{*} \in X^{*} \subset A_{u}\left(B_{X}\right)$ we have

$$
z_{\alpha}\left(x^{*}\right)=\tilde{\delta}_{z_{\alpha}}\left(x^{*}\right) \rightarrow \varphi\left(x^{*}\right)=z\left(x^{*}\right),
$$

where the last equality holds because $\varphi$ belongs to $\mathcal{M}_{z}\left(A_{u}\left(B_{X}\right)\right)$. Therefore, $z_{\alpha}$ weak-star converges to $z$. We also have

$$
\varphi(P)=\lim _{\alpha} \tilde{P}\left(z_{\alpha}\right)
$$

and these two facts show that $\varphi(P)$ belongs to $C l(P, z)$. As we observed in the introduction, $\tilde{P}(z)$ also belongs to $C l(P, z)$. Since $\left|\tilde{P}\left(z_{\alpha}\right)-\tilde{P}(z)\right| \geq \varepsilon$ for all $\alpha$, we see from (8) that $\varphi \neq \tilde{\delta}_{z}$. By [ACGLM12, Lemma 2.1], $C l(P, z)$ is a compact connected subset of $\mathbb{C}$, and hence $\operatorname{Card}(C l(P, z)) \geq c$. Now, by ACGLM12, Lemma 2.2], we have $C l(P, z) \subset \hat{P}\left(\mathcal{M}_{z}\left(A_{u}\left(B_{X}\right)\right)\right)$ and the conclusion follows.

As for the second assertion, it is observed in BoyRya98, Proposition 1] that if the restriction of a homogeneous polynomial to the open unit ball is not weakly continuous at 0 , then it is also weakly discontinuous at any point of $B_{X}$. In fact, the same argument shows that if $X=Y^{* *}$ is the bidual of a Banach space $Y$ and if the restriction, to $B_{Y^{* *}}$, of a homogeneous polynomial is weak-star discontinuous at 0 , then it is weak-star discontinuous at every point of $B_{Y^{* *}}$. Thus, since the restriction of $P$ to the open unit ball is not weakly continuous at 0 , then the restriction of $\tilde{P}$ to the open unit ball of the bidual is also weak-star discontinuous at any point of the unit ball of the bidual. 
The above remark and the Goldstine Theorem allow us to find a net $\left(z_{\alpha}\right) \subset B_{X^{* *}}$ which is weak-star convergent to $z$ but such that $\tilde{P}\left(z_{\alpha}\right)$ does not converge to $\tilde{P}(z)$. Thus, we may assume that there exists $\varepsilon>0$ such that $\left|\tilde{P}\left(z_{\alpha}\right)-\tilde{P}(z)\right| \geq \varepsilon$ for all $\alpha$. Since the net $\left(\tilde{\delta}_{z_{\alpha}}\right)$ is included in the compact set $\mathcal{M}\left(A_{u}\left(B_{X}\right)\right)$, there exists a subnet $\left(\tilde{\delta}_{z_{\beta}}\right)$ that converges to some $\varphi \in \mathcal{M}\left(A_{u}\left(B_{X}\right)\right)$. Since

$$
\varphi(f)=\lim _{\beta} \tilde{f}\left(z_{\beta}\right),
$$

for all $f \in A_{u}\left(B_{X}\right)$ we get that $\varphi\left(x^{*}\right)=z\left(x^{*}\right)$ for every $x^{*} \in X^{*}$, which means that $\varphi \in \mathcal{M}_{z}\left(A_{u}\left(B_{X}\right)\right)$.

We can again use Lemma 1.2 to obtain the corresponding result for $H_{b}(X)$.

Corollary 2.7. Let $X$ be a Banach space which admits a homogeneous continuous polynomial whose restriction to the open unit ball is not weakly continuous at 0 . Then, $\operatorname{Card}\left(\mathcal{M}_{z}\right) \geq c$ for every $z \in X^{* *}$.

The simplest examples fulfilling the hypotheses of the previous results are the spaces $\ell_{p}$ for $1 \leq p \leq \infty$. For $1<p<\infty$, the canonical basis is weakly null, so we can take the polynomial $Q(x)=\sum_{n=1}^{\infty} x_{n}^{m}$ with $m \in \mathbb{N}, m \geq p$. Moreover, in [AGGM96, Remark 1.6] it is shown that these hypotheses hold if $\ell_{2}$ is a quotient of $X$ and, as a consequence, when $X$ contains a copy of $\ell_{1}$ (for this case, see also ADR84]). This shows that $\ell_{\infty}$ and, of course, $\ell_{1}$ satisfy the hypotheses. The case $p=1$ is treated more deeply in the next section.

\section{Fibers of $\mathcal{M}\left(A_{u}\left(B_{\ell_{1}}\right)\right)$}

Many of our results describing the spectrum of $H_{b}(X)$ rely on the corresponding description of $\mathcal{M}\left(A_{u}\left(B_{X}\right)\right)$. In this section we take the study of (the fibers of) $\mathcal{M}\left(A_{u}\left(B_{\ell_{1}}\right)\right)$ a bit further. Our motivation is not only to obtain tools for the description of $\mathcal{M}\left(A_{u}\left(B_{\ell_{1}}\right)\right)$ but also to show some unexpected behavior which is interesting in itself. To put this into perspective, we begin by collecting some facts that appear, not always explicitly, in the previous sections.

First, for fibers lying over elements of the unit sphere of $\ell_{1}$, the comments before Corollary 2.4 actually show the following.

Proposition 3.1. For any $x \in S_{\ell_{1}}$, the fiber $\mathcal{M}_{x}\left(A_{u}\left(B_{\ell_{1}}\right)\right)$ is just $\left\{\delta_{x}\right\}$.

As mentioned above, $\ell_{1}$ satisfies the hypotheses of Proposition 2.6. As a consequence, $\operatorname{Card}\left(\mathcal{M}_{z}\left(A_{u}\left(B_{\ell_{1}}\right)\right)\right) \geq c$ for every $z \in B_{\ell_{1}^{* *}}$. However, much more can be said.

Proposition 3.2. If $z \in B_{\ell_{1}^{* *}}$, then $\beta \mathbb{N}$ is embedded in $\mathcal{M}_{z}\left(A_{u}\left(B_{\ell_{1}}\right)\right)$.

Proof. Let $\left\{I_{k}\right\}_{k \in \mathbb{N}}$ be a countable partition of $\mathbb{N}$ such that each $I_{k}$ is infinite and consider $\ell_{1}\left(I_{k}\right)$ as a complemented subspace of $\ell_{1}$ with basis $\left\{e_{n}: n \in I_{k}\right\}$. We have

$$
0 \in{\overline{\left\{e_{n}-e_{m}: n \neq m, n, m \in I_{k}\right\}}}^{w\left(\ell_{1}, \ell_{\infty}\right)},
$$

for every $k$.

Given $z \in B_{\ell_{1}^{* *}}$ we take $r>0$ such that $\|z\|+2 r<1$. Then, $z$ is a $w\left(\ell_{1}^{* *}, \ell_{\infty}\right)$-limit point of each of the sets

$$
\mathcal{S}_{k}=\left\{r e_{n}-r e_{m}+z: \quad n \neq m, n, m \in I_{k}\right\} \subset B_{\ell_{1}^{* *}} .
$$


The compactness of $\mathcal{M}\left(A_{u}\left(B_{\ell_{1}}\right)\right)$ allows us to find a net in $\mathcal{S}_{k}, w\left(\ell_{1}^{* *}, \ell_{\infty}\right)$ converging to $z$, whose evaluations converge to some $\varphi_{k}$ in $\mathcal{M}\left(A_{u}\left(B_{\ell_{1}}\right)\right)$. This means, in particular, that $\varphi_{k}$ is in $\mathcal{M}_{z}\left(A_{u}\left(B_{\ell_{1}}\right)\right)$ for each $k$.

Denote by $\left\{e_{k}^{*}\right\}_{k}$ the dual basic sequence of $\left\{e_{k}\right\}_{k}$. For $k \in \mathbb{N}$ we define $z_{k}=$ $z\left(e_{k}^{*}\right)$. The sequence $\left(z_{k}\right)_{k}$ is, in fact, the image of $z$ under the natural projection

$$
q: \ell_{1}^{* *}=c_{0}^{* * *} \longrightarrow c_{0}^{*}=\ell_{1} .
$$

Given $\left(a_{k}\right) \in \ell_{\infty}$, we define $P: \ell_{1} \longrightarrow \mathbb{C}$ by

$$
P(x)=\sum_{k=1}^{\infty} \frac{a_{k}}{2 r^{2}} \sum_{j \in I_{k}}\left(x_{j}-z_{j}\right)^{2},
$$

which is well defined and continuous, since $\left(z_{k}\right)_{k}$ belongs to $\ell_{1}$.

Suppose for a moment that we have shown that

$$
\tilde{P}\left(r e_{n}-r e_{m}+z\right)=a_{k}
$$

for every $n \neq m, n, m \in I_{k}$ (i.e., $\tilde{P} \equiv a_{k}$ on $\mathcal{S}_{k}$ ). Since $\varphi_{k}$ is a cluster point of the evaluations in elements of $\mathcal{S}_{k}$, we conclude that $\varphi_{k}(P)=a_{k}$. Therefore, $\left(\varphi_{k}\right)_{k=1}^{\infty}$ is an interpolating sequence contained in the compact set $\mathcal{M}_{z}\left(A_{u}\left(B_{\ell_{1}}\right)\right)$. In particular, the closure of $\left(\varphi_{k}\right)_{k=1}^{\infty}$ is topologically homeomorphic to $\beta \mathbb{N}$.

So it remains to show (10). Write

$$
\begin{aligned}
P(x) & =\sum_{k=1}^{\infty} \frac{a_{k}}{2 r^{2}} \sum_{j \in I_{k}}\left(x_{j}-z_{j}\right)^{2} \\
& =\sum_{k=1}^{\infty} \frac{a_{k}}{2 r^{2}} \sum_{j \in I_{k}} x_{j}^{2}-\sum_{k=1}^{\infty} \frac{a_{k}}{r^{2}} \sum_{j \in I_{k}} x_{j} z_{j}+\sum_{k=1}^{\infty} \frac{a_{k}}{2 r^{2}} \sum_{j \in I_{k}} z_{j}^{2} \\
& =P_{2}(x)-P_{1}(x)+P_{0} .
\end{aligned}
$$

The linear part is easy to handle:

$$
\tilde{P}_{1}\left(r e_{n}-r e_{m}+z\right)=\sum_{k=1}^{\infty} \frac{a_{k}}{r^{2}} \sum_{j \in I_{k}}\left(r \delta_{n, j}-r \delta_{m, j}+z_{j}\right) z_{j} .
$$

For the 2-homogeneous part, note that $P_{2}$ is a diagonal polynomial, so Example 1.2 in [LlaMor04] shows that $\tilde{P}_{2}=P_{2} \circ q$, where $q$ is defined in (9). This means that

$$
\tilde{P}_{2}\left(r e_{n}-r e_{m}+z\right)=\sum_{k=1}^{\infty} \frac{a_{k}}{2 r^{2}} \sum_{j \in I_{k}}\left(r \delta_{n, j}-r \delta_{m, j}+z_{j}\right)^{2} .
$$

Combining this information, for $m, n \in I_{l}, m \neq n$, we obtain

$$
\begin{aligned}
\tilde{P}\left(r e_{n}-r e_{m}+z\right)= & \sum_{k=1}^{\infty} \frac{a_{k}}{2 r^{2}} \sum_{j \in I_{k}}\left(r \delta_{n, j}-r \delta_{m, j}+z_{j}\right)^{2} \\
& -\sum_{k=1}^{\infty} \frac{a_{k}}{r^{2}} \sum_{j \in I_{k}}\left(r \delta_{n, j}-r \delta_{m, j}+z_{j}\right) z_{j}+\sum_{k=1}^{\infty} \frac{a_{k}}{2 r^{2}} \sum_{j \in I_{k}} z_{j}^{2} \\
= & \sum_{k=1}^{\infty} \frac{a_{k}}{2 r^{2}} \sum_{j \in I_{k}}\left(r \delta_{n, j}-r \delta_{m, j}\right)^{2}=\frac{a_{l}}{2 r^{2}} \sum_{j \in I_{l}}\left(r \delta_{n, j}-r \delta_{m, j}\right)^{2}=a_{l},
\end{aligned}
$$

which ends the proof. 
Finally we study fibers above elements of the unit sphere of the bidual of $\ell_{1}$. In Proposition 3.1 we have seen that fibers over the unit sphere of $\ell_{1}$ are trivial. Let us see that this is not the case for a large number of elements in $S_{\ell_{1}^{* *} \text {. }}$

Proposition 3.3. There exists a copy $\mathcal{K}$ of $\beta \mathbb{N}$ in $S_{\ell_{1}^{* *}}$ such that, for each $z \in \mathcal{K}$, the cardinal of the fiber $\mathcal{M}_{z}\left(A_{u}\left(B_{\ell_{1}}\right)\right)$ is at least $c$.

Proof. As before, take $\left\{I_{k}\right\}_{k \in \mathbb{N}}$ a countable partition of $\mathbb{N}$ such that each $I_{k}$ is infinite. Now, for each $k$, consider $z^{k} \in \bar{B}_{\ell_{1}}^{* *}$, a weak-star cluster point of $\left(e_{n}\right)_{n \in I_{k}}$. It is easy to see that $\left(z^{k}\right)_{k}$ is an interpolating sequence in $\bar{B}_{\ell_{1}^{* *}}$ : given $a=\left(a_{k}\right)_{k} \in \bar{B}_{\ell_{\infty}}$, we define $x_{a}^{*} \in \bar{B}_{\ell_{1}^{*}}$ by $x_{a}^{*}\left(e_{j}\right)=a_{k}$, if $j \in I_{k}$. Then, $z^{k}\left(x_{a}^{*}\right)=a_{k}$, for all $k$. As a consequence, the closure $\mathcal{K}$ of $\left(z^{k}\right)_{k}$ in $\bar{B}_{\ell_{1}}^{* *}$ (with the weak-star topology) is homeomorphic to $\beta \mathbb{N}$.

Now, given $\overrightarrow{1}=(1, \ldots, 1, \ldots) \in \bar{B}_{\ell_{1}^{*}}$ we have $z^{k}(\overrightarrow{1})=1$ for all $k$, and so $z(\overrightarrow{1})=1$ for all $z \in \mathcal{K}$. Therefore, $\mathcal{K}$ is a weak-star compact subset of $S_{\ell_{1}^{* *}}$.

It remains to show that fibers over elements in $\mathcal{K}$ are big. Given $z \in \mathcal{K}$, note that

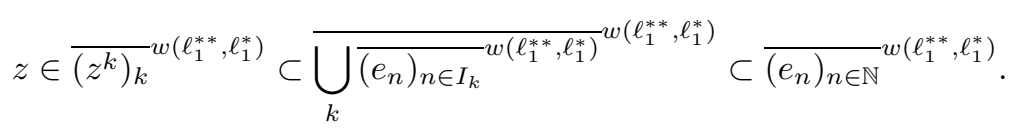

This means that $z$ is the weak-star limit of a subnet $\left(e_{n_{\alpha}}\right)_{\alpha}$ of $\left(e_{n}\right)_{n}$. If $\varphi$ is a limit point of $\left(\delta_{e_{n_{\alpha}}}\right)_{\alpha}$ in $\mathcal{M}\left(A_{u}\left(B_{\ell_{1}}\right)\right)$, we see as in Proposition 2.5 that $\varphi$ belongs to $\mathcal{M}_{z}\left(A_{u}\left(B_{\ell_{1}}\right)\right)$ and that $\varphi \neq \tilde{\delta}_{z}$.

The rest of the argument follows the lines of the proof of Proposition 2.6. Take $f$ such that $\varphi(f) \neq \tilde{f}(z)$. Both numbers belong to the connected set $C l(f, z)$, so that $\operatorname{Card}(C l(f, z)) \geq c$. Appealing to [ACGLM12, Lemma 2.2], we have that

$$
C l(f, z) \subset \hat{f}\left(\mathcal{M}_{z}\left(A_{u}\left(B_{X}\right)\right)\right),
$$

and the conclusion follows.

Let us summarize what we know and what the results of this section tell us about the spectrum $\mathcal{M}\left(H_{b}(X)\right)$ for $X=\ell_{1}$. As usual, we translate our results for $\mathcal{M}\left(A_{u}\left(B_{\ell_{1}}\right)\right)$ to $\mathcal{M}\left(H_{b}\left(\ell_{1}\right)\right)$ by means of Lemma 1.2 .

Theorem 3.4. Let $X=\ell_{1}$.

(a) If $x \in \ell_{1}$, the only element $\varphi \in \mathcal{M}_{x}$ with $R(\varphi)=\|x\|$ is $\delta_{x}$. In other words,

$$
\mathcal{M}_{x,\|x\|}=\left\{\delta_{x}\right\} .
$$

(b) For each $r$, there is a copy $\mathcal{K}$ of $\beta \mathbb{N}$ in $r S_{\ell_{1}^{* *}}$ such that for each $z \in \mathcal{K}$,

$$
\operatorname{Card}\left(\mathcal{M}_{z,\|z\|}\right) \geq c .
$$

(c) For every $z \in \ell_{1}^{* *}$ and every $\varepsilon>0$, the fiber $\mathcal{M}_{z,\|z\|+\varepsilon}$ contains a copy of $\beta \mathbb{N}$.

Proof. Statement (a) is just Corollary 2.4, restated as in (7). Item (b) follows from Proposition 3.3 and Lemma 1.2. Note that these elements $z$ must necessarily belong to $\ell_{1}^{* *} \backslash \ell_{1}$. Finally, statement (c) follows from Proposition 3.2 and Lemma 1.2 . 


\section{ACKNOWLEDGEMEnTS}

The authors thank the anonymous referee for valuable suggestions and comments that improved this paper. Part of this work was developed during the visits of the first, second and third authors to the Departament d'Anàlisi Matemàtica of the Universitat de València. They thank the department and its members for their kind hospitality.

\section{REFERENCES}

[AcoLou07] María D. Acosta and Mary Lilian Lourenço, Shilov boundary for holomorphic functions on some classical Banach spaces, Studia Math. 179 (2007), no. 1, 27-39, DOI 10.4064/sm179-1-3. MR2291721(2008c:46061)

[AroBer78] Richard M. Aron and Paul D. Berner, A Hahn-Banach extension theorem for analytic mappings (English, with French summary), Bull. Soc. Math. France 106 (1978), no. 1, 3-24. MR508947 (80e:46029)

[ACG91] R. M. Aron, B. J. Cole, and T. W. Gamelin, Spectra of algebras of analytic functions on a Banach space, J. Reine Angew. Math. 415 (1991), 51-93. MR.1096902 (92f:46056)

[ACGLM12] Richard M. Aron, Daniel Carando, T. W. Gamelin, Silvia Lassalle, and Manuel Maestre, Cluster values of analytic functions on a Banach space, Math. Ann. 353 (2012), no. 2, 293-303, DOI 10.1007/s00208-011-0681-0. MR2915537

[ADR84] R. M. Aron, J. Diestel, and A. K. Rajappa, Weakly continuous functions on Banach spaces containing $l_{1}$, Banach spaces (Columbia, Mo., 1984), Lecture Notes in Math., vol. 1166, Springer, Berlin, 1985, pp. 1-3, DOI 10.1007/BFb0074685. MR827751 (87g:46022)

[AGGM96] R. M. Aron, P. Galindo, D. García, and M. Maestre, Regularity and algebras of analytic functions in infinite dimensions, Trans. Amer. Math. Soc. 348 (1996), no. 2, 543-559, DOI 10.1090/S0002-9947-96-01553-X. MR1340167 (96g:46032)

[BoyRya98] C. Boyd and R. A. Ryan, Bounded weak continuity of homogeneous polynomials at the origin, Arch. Math. (Basel) 71 (1998), no. 3, 211-218, DOI 10.1007/s000130050254. MR1637369 (99k:46076)

[CGMS12] Daniel Carando, Domingo García, Manuel Maestre, and Pablo Sevilla-Peris, On the spectra of algebras of analytic functions, Topics in complex analysis and operator theory, Contemp. Math., vol. 561, Amer. Math. Soc., Providence, RI, 2012, pp. 165198, DOI 10.1090/conm/561/11114. MR2905544(2012k:46001)

[Car62] Lennart Carleson, Interpolations by bounded analytic functions and the corona problem, Ann. of Math. (2) 76 (1962), 547-559. MR0141789 (25 \#5186)

[ColLoh66] E. F. Collingwood and A. J. Lohwater, The theory of cluster sets, Cambridge Tracts in Mathematics and Mathematical Physics, No. 56, Cambridge University Press, Cambridge, 1966. MR0231999(38 \#325)

[DavGam89] A. M. Davie and T. W. Gamelin, A theorem on polynomial-star approximation, Proc. Amer. Math. Soc. 106 (1989), no. 2, 351-356, DOI 10.2307/2048812. MR947313 (89k:46023)

[Die84] Joseph Diestel, Sequences and series in Banach spaces, Graduate Texts in Mathematics, vol. 92, Springer-Verlag, New York, 1984. MR737004 (85i:46020)

[Din99] Seán Dineen, Complex analysis on infinite-dimensional spaces, Springer Monographs in Mathematics, Springer-Verlag London, Ltd., London, 1999. MR.1705327 (2001a:46043)

[Far98] Jeff D. Farmer, Fibers over the sphere of a uniformly convex Banach space, Michigan Math. J. 45 (1998), no. 2, 211-226, DOI 10.1307/mmj/1030132179. MR.1637638 (99h:46063)

[Gam69] Theodore W. Gamelin, Uniform algebras, Prentice-Hall, Inc., Englewood Cliffs, N. J., 1969. MR0410387 (53 \#14137)

[Gam94] Theodore W. Gamelin, Analytic functions on Banach spaces, Complex potential theory (Montreal, PQ, 1993), NATO Adv. Sci. Inst. Ser. C Math. Phys. Sci., vol. 439, Kluwer Acad. Publ., Dordrecht, 1994, pp. 187-233. MR.1332962 (96m:46082) 
[JohOrt12a] W. B. Johnson and S. Ortega Castillo, The cluster value problem in spaces of continuous functions, Proc. Amer. Math. Soc. 143 (2015), no. 4, 1559-1568, DOI 10.1090/S0002-9939-2014-12190-3. MR3314069

[JohOrt12b] W. B. Johnson and S. Ortega Castillo, The cluster value problem for Banach spaces, preprint.

[LlaMor04] J. G. Llavona and L. A. Moraes, The Aron-Berner extension for polynomials defined in the dual of a Banach space, Publ. Res. Inst. Math. Sci. 40 (2004), no. 1, 221-230. MR2030074(2004k:46069)

[Muj86] Jorge Mujica, Complex analysis in Banach spaces, Holomorphic functions and domains of holomorphy in finite and infinite dimensions, Notas de Matemática [Mathematical Notes], 107, North-Holland Mathematics Studies, vol. 120, North-Holland Publishing Co., Amsterdam, 1986. MR842435 (88d:46084)

[Munkres] J. M. Munkres, Topology, Second Edition. Prentice Hall, Upper Saddle River, 2000. [Sch61] I. J. Schark, Maximal ideals in an algebra of bounded analytic functions, J. Math. Mech. 10 (1961), 735-746. ("I. J. Schark" is a pseudonym for the group: Irving Kaplansky, John Wermer, Shizuo Kakutani, R. Creighton Buck, Halsey Royden, Andrew Gleason, Richard Arens and Kenneth Hoffman.) MR.0125442 (23 \#A2744)

Department of Mathematical Sciences, Kent State University, Kent, Ohio 44242

E-mail address: aron@math.kent.edu

Departamento de Matemática, Pab i, Facultad de Ciencias Exactas y Naturales, Universidad de Buenos Aires, (1428) Buenos Aires, Argentina - And - IMAS - COniCET

E-mail address: dcarando@dm.uba.edu

Departamento de Matemática, Universidad de San Andrés, Vito Dumas 284, (B1644BID) Victoria, Buenos Aires, Argentina - And - IMAS - CONICET

E-mail address: slassalle@udesa.edu.ar

Departmento de Análisis Matemático, Universidad de Valencia, Doctor Moliner 50, 6100 Burjasot (VAlencia), Spain

E-mail address: Manuel. Maestre@uv.es 